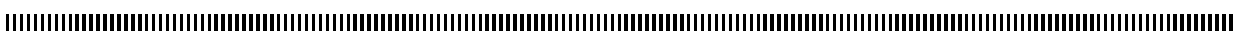

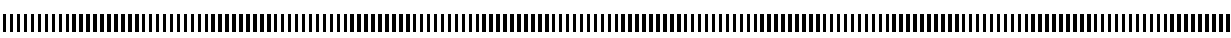
| |

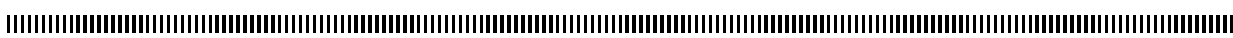
| | | |

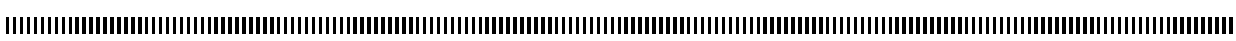

\title{
Ockham's razor: Deriving cyclic evolutions from viability and inertia constraints
}

\author{
Jean-Pierre Aubin \\ LASTRE (Laboratoire d'Applications des Systèmes Tychastiques Régulés) \\ 14, rue Domat F-75005 Paris \\ aubin.jp@gmail.com
}

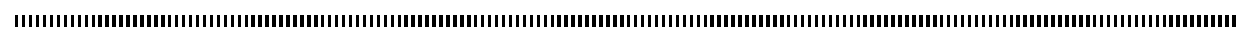

ABSTRACT. This article deals with a theme to which Claude Lobry has been interested for a long time: what is the nature of mathematics motivated by biological sciences? It starts by presenting the subjective opinions of its author, illustrated by the simplest application one can think of: demonstrating that it is possible to produce cyclic evolutions on the simple basis of viability and inertia constraints, without using periodic differential equations. It is not impossible that this approach is foreign to an explanation of biological clocks (or economic cycles in another field).

RÉSUMÉ. Cet article traite d'un thème auquel Claude Lobry s'est longtemps intéressé : quelle est la nature des mathématiques motivées par les sciences biologiques ? II commence par exposer les opinions subjectives de l'auteur, illustrées pas une application des plus simples démontrant qu'il est possible de produire des évolutions cycliques à partir de simples hypothèses (contraintes de viabilité et d'inertie) pour produire des évolutions cycliques, sans faire appel aux équations différentielles périodiques. II n'est pas impossible que ce point de vue soit étranger à une explication du fonctionnement des horloges biologiques (ou des cycles économiques dans un autre domaine).

KEYWORDS : Viability, Biomathematics, Cyclicity, Inverse Problems, Hysteresis, Inertia, Inertia Function, Inertia Principle, Punctuated Equilibrium, Viability Kernels

MOTS-CLÉS : Viabilité, Biomathématiques, Cyclicité, Problèmes Inverse, Hystérèse, Inertie, Fonction d'inertie, Principe d'inertie, Équilibres Ponctués, Noyaux de Viabilité

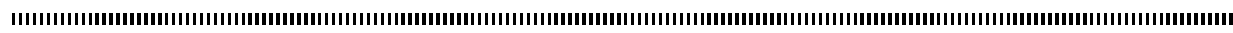




\begin{abstract}
À Claude Lobry, que j'imagine bougonnant, grommelant, ronchonnant, maugréant, grognant, pestant

à la lecture de ces pages, cherchant quels pourraient être les points de désaccord sur un sujet longtemps débattu entre nous, en lui souhaitant, en toute amitié, d'apprécier la liberté tant attendue d'être enfin son propre esclave.
\end{abstract}

\title{
1. Introduction
}

What are the mathematical tools most useful for understanding "living” systems? I do not know how to answer this question, far too ambitious, but except by saying that,

1) even if some of these tools do exist, they have been mostly motivated by the observation of the inert (physical) systems,

2) these tools and methods have to be analyzed to check whether their use may change the formulation of the question,

3 ) and not the other way around, except when aware of the dangers and assessing them,

4) new tools must be forged and emerge to be selected by "cognitive evolution", with a ... Darwinian flavor, minimizing religion wars on such or such approach.

I can at least bring a personal definitively subjective negative answer to the the subsidiary question whether living systems are sufficiently similar to systems currently studied in mathematics, physics, or engineering. Eugene Wigner's considerations on the unreasonable effectiveness of mathematics in the natural sciences [22, Wigner] are even more relevant in living sciences.

Whenever I am asked to define what are mathematics, I am tempted to cite Saint Augustine's confession: 'If no one asks me, I know: if I wish to explain it to one that asketh, I know not: yet I say boldly that I know, ..." he wrote (about time) in the book XI (11.14.17) of his Confessions.

For at least one good reason, since "mathematics" mean both collections of mathematical results at a given time and mathematical "cognitive processes". Mathematics as end-results are as many as mathematicians, whereas one may find some regularities in mathematical cognitive processes.

For many centuries, human minds used their potential "mathematical capabilities" to describe and share their "mathematical perceptions" of the world. This mathematical capability of human brains is assumed to be analogous to the language capability. Each child coming to this world uses this specific capability in social interaction with other people to reach (evolving) consensus on the perception of their world by learning their mother tongue (and few others before this capability fades away with age). I suggest the same happens with mathematics. They play the "mathematical role" of metaphors that language uses for allowing us to understand a new phenomenon by metaphors comparing it with previously "understood phenomena". Before it exploded recently in a Babel skyscraper, 
this "mathematical father tongue" was quite consensual and perceived as universal. This is this very universality which makes mathematics so fascinating, deriving mathematical theories or tools motivated by one field to apply them to several other ones. However, apparently, because up to now, the mathematical "father tongue" was mainly shaped by "simple" physical problems of the inert part of the environment, letting aside, with few exceptions, the living world. For good reasons. Basic simple principles, such as the Pierre de Fermat's "variational principle", including Isaac Newton's law thanks to Maupertuis's least action principle, derived explanations of complex phenomena from simple principles, as Ockham's razor prescribes: This "law of parsimony" states that an explanation of any phenomenon should make as few assumptions as possible, and to choose among competing theories the one that postulates the fewest concepts. This is the result of an "abstraction process", which is the (poor) capability of human brains that selects among the perceptions of the world the few ones from which they may derive logically or mathematically many other ones. Simplifying complexity should be the purpose of an emerging science of complexity, if such a science will emerge beyond its present fashionable status. "If people do not believe that mathematics is simple, it is only because they do not realize how complicated life is", said John von Neumann.

Biology, for instance, offers to our investigations myriads of biological clocks or oscillators, producing periodic evolutions, or, rather, cyclic evolutions (see [23, Winfree], [13. Lobry], for instance, and more recently, [12, Francoise]). This modification of the terminology is justified by the fact that nowadays, periodic evolutions are understood as produced by a system of differential equations. The search of these equations is a very difficult undertaking, so that the question arises to look for other ways to produce periodic solutions, that we suggest to call cyclic to underlie the fact that they are not periodic solutions of a given system of differential equations. As often in biology or in life sciences, we face the following dilemma:

1 [Simple Dynamics and Complex Constraints] Are the examples of biological clocks produced by complex systems of differential equations with complex coefficients or produced by very simple dynamics, confronted to a complex maze of constraints?

This may be how Ockham's razor can apply in this domain, and this paper attempts to prove this point, because, in my subjective view, the right hand sides of these complex systems are actually unknown or poorly known to us. Cyclic evolutions can alternatively be derived from non periodic control systems, subjected to "viability constraints" and "inertia thresholds", as we shall see.

Remark - For example, the history of the mathematical modeling of the propagation of the nervous influx started in 1903 with very simple impulse models by Louis Lapicque. At the time, a mathematical theory of impulse systems did not exist yet. This triggered the search of systems of differential equations reproducing evolutions "looking like" or reminiscent of the propagation or the nervous influx by the ingenious works of Alan Hodgkin and Andrew Huxley (nephew of the writer Aldous Huxley and grandson of Thomas Huxley). These equations reproduce evolutions looking like nervous influxes, but without producing explanations, whereas the Lapicque model, very simple indeed, but 
involving constraints and impulse evolutions when they reach those constraints, provides both a simple explanation and evolutions looking like the propagation of the nervous influx.

So physics, which could be defined as the part of the cultural environment which is understandable by mathematical metaphors, has not yet, in my opinion, encapsulated the mathematical metaphors of living systems, from organic molecules to social systems made of human brains. The reason seems to be that the adequate mathematical tongue does not yet exist. And the challenge is that before creating it, the present one has to be forgotten, de-constructed. This is quite impossible because mathematicians have been educated in the same way all over the world, depriving mathematics from the Darwinian evolution which has operated on languages. This uniformity is the strength and the weakness of present day mathematics: Its universality is partial. The only possibility to perceive mathematically living systems will remain a dream: To gather in secluded convents young children with good mathematical capability, but little training in the present mathematics, under the supervision or guidance of economists or biologists without mathematical training. They possibly could come up with new mathematical languages unknown to us providing the long expected unreasonable effectiveness of mathematics in the economic and biological sciences.

Even the concept of natural number is oversimplifying, by putting in a same equivalence class so several different sets, erasing their qualitative properties or hiding them behind their quantitative ones. Numbers, next measurements, and then, statistics, how helpful they are for understanding and controlling the physical part of the environment, may be a drawback to address the qualitative aspects of our world, left to plain language for the quest of elucidation. We may have to return to the origins and explore new "qualitative" routes, without overusing the mathematics that our ancestors accumulated so far and bequeathed to us.

Meanwhile, we are left with this paradox: "simple" physical phenomena are explained by more and more sophisticated and abstract mathematics, whereas "complex" phenomena of living systems use, most of the time, relatively rudimentary mathematical tools.

For instance, in economics, the mathematical tools used so far did not answer the facts that, for instance,

1) economic evolution is never at equilibrium (stationary state),

2) and thus, there were no need that it converges to it, in a stable or unstable way,

3) that elementary cognitive sciences cannot accept the rationality assumption of human brains,

4) and even more that they can be reduced to utility functions, the existence of which was already questioned by Henri Poincaré when he wrote to Léon Walras that "Satisfaction is thus a magnitude, but not a measurable magnitude" (numbers are not sufficient to grasp satisfaction),

5) that uncertainty can be mathematically captured only by probabilities (numbers, again),

6) that chaos, defined as a property of deterministic system, is not fit to represent a nondeterministic behavior of living systems which struggle to remain as stable (and thus, "non chaotic") as possible, 
7) that intertemporal optimality, a creation of the human brain to explain some physical phenomena, is not the only creation of Nature, (in the sense that "Nature" created it only through human brains!),

8) that those human brains should complement it by another and more recent principle, adaptation of transient evolutions to environments,

9) and so on (see for instance [3, Aubin]).

These considerations are developed in [6, Aubin]. The purpose of viability "theory" (in the sense of a sequence [theôria, procession] of mathematical tools sharing a common background, and not necessarily an explanation of something [theôrein, to observe]), is to attempt to answer directly the question of dynamic adaptation of uncertain evolutionary systems to environments defined by constraints, that we called viability constraints for obvious reasons. Hence the name of this body of mathematical results developed since the end of the 1970's that needed to forge a differential calculus of set-valued maps (set-valued analysis, see [9, Aubin\& Frankowska]), differential inclusions and differential calculus in metric spaces (mutational analysis and morphogenesis, see [10, Aubin \& Lesne] and [4, Aubin]). These results, how imperfect they might be to answer this challenge, have at least been motivated by social and biological sciences, even though constrained by the mathematical training of their authors. See details in [1, Aubin] and [7. Aubin, Bayen, Bonneuil \& Saint-Pierre].

\section{Chance and Necessity}

It is by now a consensus that the evolution of many variables describing systems, organizations, networks arising in biology and human and social sciences do not evolve in a deterministic way, and in many instances, not even in a stochastic way as it is usually understood, but with a Darwinian flavor.

Viability theory started in 1976 by translating mathematically the title

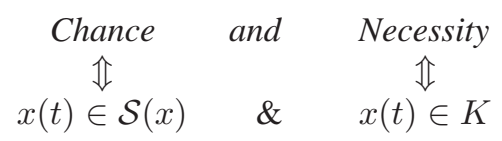

of the famous 1973 book by [19, Jacques Monod], taken from an (apocryphical?) quotation of Democritus who held that "the whole universe is but the fruit of two qualities, chance and necessity”.

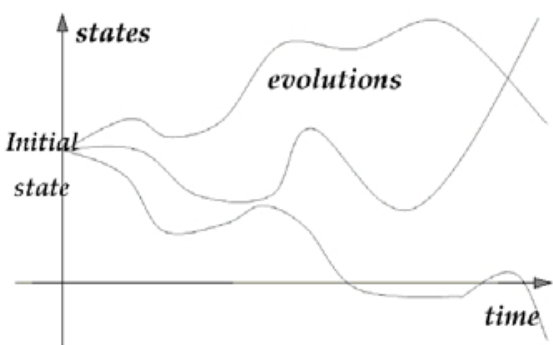

2 The mathematical translation of "chance". The mathematical translation of "chance" is a kind of evolutionary engine, called an evolutionary system, associating with any initial state $x$ the subset $\mathcal{S}(x)$ of evolutions starting at $x$.

The figure displays evolutions starting from a given initial state, which are functions from time (in abscissas) to the state space (ordinates). 
The system is said to be deterministic if for any initial state $x, \mathcal{S}(x)$ is made of one and only one evolution, whereas "contingent uncertainty" happens when the subset $\mathcal{S}(x)$ of evolutions contains more than one evolution for at least one initial state.

Note that the present evolves, too, so that initial conditions do evolve in accordance. Therefore, this definition of evolutionary systems should depend upon a flow of past initial conditions and on the (history of the) evolutions.

"Contingence is a non-necessity, it is a characteristic attribute of freedom", wrote Gottfried Leibniz.

The mathematical transla-
tion of "necessity". The mathe-
matical translation of "necessity"
is the requirement that for all $t \geq$
$0, x(t) \in K$, meaning that at each
instant, "viability constraints" are
satisfied by the state of the sys-
tem.

In summary, the environment is described by viability constraints of various kinds, a word encompassing polysemous concepts as stability, confinement, homeostasis, adaptation, etc., expressing the idea that some variables must obey some constraints (representing physical, social, biological and economic constraints, etc.) that can never be violated. So, viability theory started as the confrontation of evolutionary systems governing evolutions and viability constraints that such evolutions must obey.

Presented in such an evolutionary perspective, this approach of (complex) evolutionary systems departs from main stream modeling by a direct approach:

4 [Direct Approach.] It consists in studying properties of evolutions governed by an evolutionary system: Gather the larger number of properties of evolutions starting from each initial state. It may be an information both costly and useless, since our brains cannot handle simultaneously too many observations and concepts.

Moreover, it may happen that

1) evolutions starting from a given initial state satisfy properties which are lost by evolutions starting from another initial state, even close to it (sensitivity analysis),

2) or that, even if all evolutions share a given set of properties, they fade away for neighboring systems (stability analysis). 
Viability theory rather uses instead an inverse approach:

5 [Inverse Approach.] A set of prescribed properties of evolutions being given, study the (possibly empty) subsets of initial states from which

1) starts at least one evolution governed by the evolutionary system satisfying the prescribed properties,

2) all evolutions starting from it satisfy these prescribed properties.

These two subsets coincide whenever the evolutionary system is deterministic.

Stationarity, periodicity and asymptotic behavior are examples of classical properties motivated by physical sciences. They are studied in terms of such parameters, as in bifurcation theory, catastrophe theory, chaotic behavior, etc., as Stephen Wiggins observed in section 3.3 "On the Interpretation and Application of Bifurcation Diagrams: A Word of Caution" of his book Introduction to nonlinear systems and chaos by : . "At this point, we have seen enough examples so that it should be clear that the term bifurcation refers to the phenomenon of a system exhibiting qualitatively new dynamical behavior as parameters are varied. However, the phrase "as parameters are varied" deserves careful consideration... In all of our analyses thus far the parameters have been constant. The point is that we cannot think of the parameter as varying in time, even though this is what happens in practice. Dynamical systems having parameters that change in time (no matter how slowly!) and that pass through bifurcation values often exhibit behavior that is very different from the analogous situation where the parameters are constant."

The point raised by Wiggins is revisited extensively in both contexts, mathematics and interpretation, in two papers of this volume : [11, Fruchard \& Schäfke], [14, Lobry]. For applications of this type of techniques, I refer to [20, Murray] and [12, Françoise] among a multitude of books and papers on this topic.

As far as chaos, a polysemous word, is concerned, it was already announced in 1750 , in connection with connectionism, by Henri Thiry, baron d'Holbach (1694-1778) in his Système de la nature: "Finally, if everything in nature is linked to everything, if all motions are born from each other although they communicate secretely to each other unseen from us, we must hold for certain that there is no cause small enough or remote enough which sometimes does not bring about the largest and the closest effects on us. The first elements of a thunderstorm may gather in the arid plains of Lybia, then will come to us with the winds, make our weather heavier, alter the moods and the passions of a man of influence, deciding the fate of several nations."

The use of chaos is reminescent, mimicking but not explaining the fact that some nonlinear differential equations produce chaotic behavior, quite sensitive to initial conditions.

Was chance rooted in deterministic system? Is uncertainty observed in living systems consistent with differential equations?

However, for many problems arising in biological, cognitive, social and economic sciences, I believe we face a completely orthogonal situation, governed by differential 
inclusions, but producing evolutions as regular or stable (in a very loose sense) as possible for the sake of adaptation and viability required for life.

Are attractors (again, defined in several ways) relevant for living systems? Do we have to restrict evolution to stationarity (at equilibrium) and to asymptotic behavior (at attractors)? Living systems provide transient evolutions, doomed to dye in finite time as soon as the are born, long before infinity. "In the long term, we are all dead" wrote Keynes.

Why not keep the underlying concept of "viability" (which coincides with invariance for deterministic systems) without its asymptotic component which is not relevant for living organisms which are doomed to die or to be transformed as soon as they are born. Furthermore, evolutions starting outside never reach the attractor in finite time!

The situation when coefficients are kept constant is familiar in physics, but, in engineering as well as in economic and biological sciences, they may have to vary with time, playing the roles of controls in engineering, of regulons in social and biological sciences, or tyches, when they play the role of random variables whenever uncertainty does not obey statistical regularity, or is subject to "extreme events".

We thus have to add to this list of classical properties other ones, such as concepts of viability of an environment, of capturability of a target in finite time, and of other concepts combining properties of this kind.

\section{Regulated Systems}

The main examples of evolutionary systems are provided by

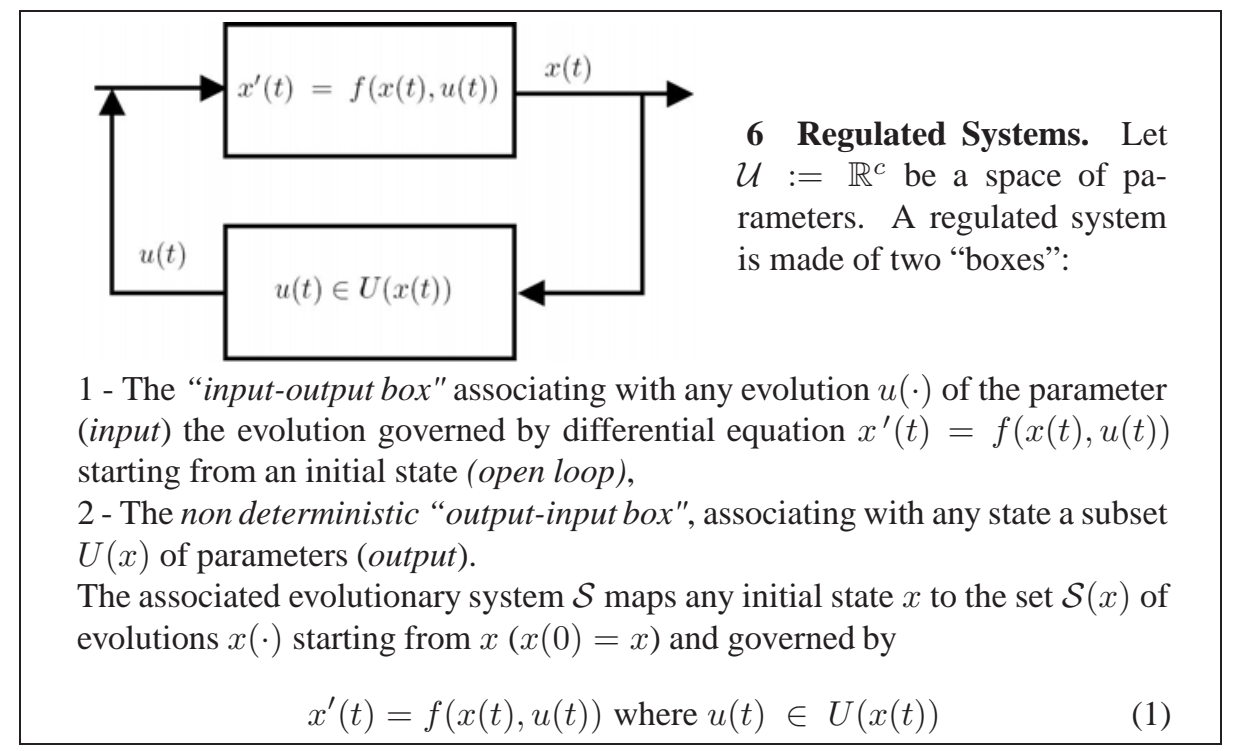

The parameters range over a state-dependent contingent map $U: x \leadsto U(x)$, providing the system opportunities to adapt at each state to viability constraints.

The nature of the parameter differs according to the problems and to questions asked: 
They can be

- "controls", whenever a controller or a decision maker "pilots" the system by choosing the controls, as in engineering,

- "regulons" or regulatory parameters in those living systems where no identified or consensual agent acts on parameters of the system,

- "tyches" or disturbances, perturbations under which nobody has any control or influence.

To be more explicit, we have to introduce some formal definitions describing the concepts of viability kernel of an environment:

Definition 3.1 [Viability Kernel] If a subset $K \subset \mathbb{R}^{d}$ is regarded as an environment (defined by viability constraints), an evolution $x(\cdot)$ is said to be viable in the environment $K \subset \mathbb{R}^{d}$ if for every time $t \geq 0, x(t)$ belongs to $K$. The viability kernel of $K$ under the evolutionary system $\mathcal{S}$ is the set $\operatorname{Viab}_{\mathbb{1}}(K)$ of initial states $x \in K$ from which starts at least one evolution $x(\cdot) \in \mathcal{S}(x)$ viable in $K$ :

$$
\operatorname{Viab}_{\mathbb{Z}}(K):=\left\{x_{0} \in K \mid \exists x(\cdot) \in \mathcal{S}\left(x_{0}\right) \text { such that } \forall t \geq 0, x(t) \in K\right\}
$$

Two extreme situations deserve to be singled out: The environment is said to be

1) viable under (Z) if it is equal to its viability kernel: $\operatorname{Viab}_{\square}(K)=K$,

2) a repeller under (1) if it is empty: $\operatorname{Viab}_{17}(K)=\emptyset$.

It is equivalent to say that all evolutions starting from a state belonging to the complement of viability kernel in $K$ leave the environment in finite time.

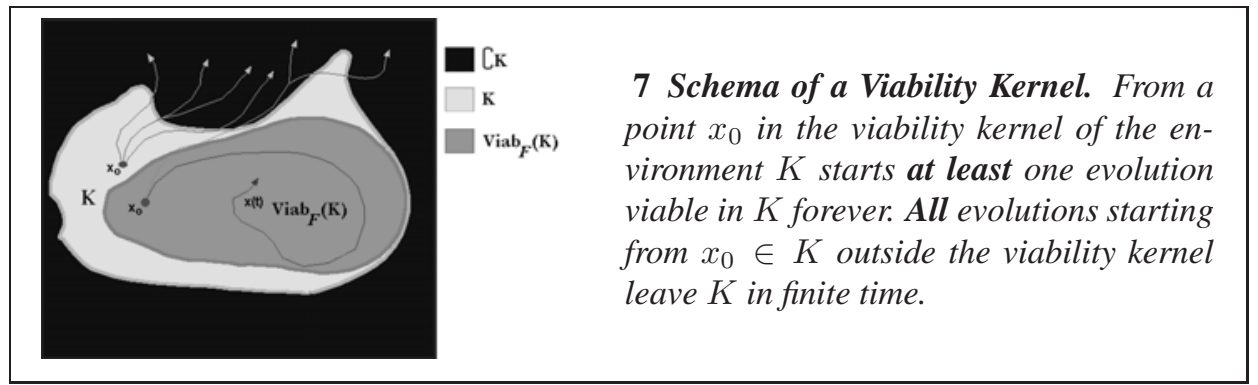

Hence, the viability kernel plays the role of a viabilimeter, the "size" of which measuring the degree of viability of an environment, so to speak. There are many other concepts or viability constraints as Hofbauer and Sigmund concept or permanence, close to the concept of persistence (see [15, Lobry \& Sari] for instance).

The main results of viability theory characterize and study, under adequate assumptions, the mathematical and algorithmic properties of the viability kernel (as well as softwares computing it), and above all, provides the regulation map regulating the viable evolutions. On the viability kernel, the regulation map associates a nonempty subset $R(x) \subset U(x)$ of regulons such that the new regulated systems

$$
x^{\prime}(t)=f(x(t), u(t)) \text { where } u(t) \in R(x(t))
$$


governs viable evolutions.

Arises next the question: How can we choose the controls or the regulons? It triggers another question: For what purpose?

The most natural one for our brains is for choosing the best one. This is the approach initiated by Fermat in optics, the prototype of variational principles in physics and by Maupertuis in celestial mechanics, which became optimal control problems in engineering.

\section{Intertemporal Optimization}

In a dynamical perspective, the main mathematical tool commonly used is optimal control theory, which deals with minimization problem of an intertemporal criterion by evolutions governed by a regulated system. This approach makes implicit requirements that deserve to be made explicit:

8 [Implicit Prerequisites for Intertemporal Optimization] Intertemporal optimization demands

1) the existence of an actor (agent, decision-maker, controller, etc.),

2) an optimality criterion,

3) that decisions are taken once and for all at the initial time,

4) a knowledge of the future (or of its anticipation).

For systems involving living beings, there is not necessarily an actor governing the evolution of regulons according to the above prerequisites. The choice of criteria is open to question even in static models, even when multicriteria or several decision makers are involved in the model. Furthermore, the choice (even conditional) of the optimal controls is made once and for all at some initial time, and thus cannot be changed at each instant so as to take into account possible modifications of the environment of the system, thus forbidding adaptation to viability constraints.

The intertemporal criterion involving the knowledge of the state at future times requires some knowledge of the future. Most living systems display a myopic behavior. Instead of taking into account the future, their evolutions are certainly constrained by their history. The knowledge of the future needs to assume some regularity (for instance, periodicity, cyclicity) of the phenomena (as in mechanics), or to make anticipations, or demands experimentation. Experimentation, by assuming that the evolution of the state of the system starting from a given initial state for a same period of time will be the same whatever the initial time, allows one to "translate" the time interval back and forth, and, thus, to "know" the future evolutions governed by the system. But in life sciences as well as in economics, the systems are irreversible, their dynamics may disappear and cannot be recreated, forbidding any insight into the future. 
Hence, we are left to forecast, predict or anticipate the future, i.e., extrapolate past evolutions and to constrain in the last analysis the evolution of the system as a function of its history. However, to quote Paul Valéry, "Forecasting is a dream from which reality wakes us up". After all, in biological evolution, intertemporal optimization can be traced back to Sumerian mythology which is at the origin of Genesis: one Decision-Maker, deciding what is good and bad and choosing the best (fortunately, on an intertemporal basis with infinite horizon, thus wisely postponing to eternity the verification of optimality), knowing the future, and having taken the optimal decisions, well, during one week...

We had to wait for Alfred Wallace to question this view in 1858 in his essay On the Tendency of Varieties to Depart Indefinitely from Original Type which he sent to Darwin who had been working on his celebrated Origin of Species (1859) since 1844. Selection by viability and not by intertemporal optimization motivated viability theory.

\section{Punctuated Equilibria and the Inertia Principle}

In the absence of an actor piloting the regulons, or by assuming that this actor is $m y$ opic, lazy, opportunistic and conservative, we cannot assume any longer that the regulons are chosen to minimize an intertemporal criterion. We may assume instead that regulons evolve as "slowly" as possible because the change of regulons (or controls in engineering) is costly, even very costly.

Evolutions under constant coefficients, which do not evolve at all, may not satisfy required properties, such as viability, capturability or optimality. Then the question arises to study when, where and how coefficients must cease to be constant and start to "evolve" in order to guarantee the viability property, for instance. In this case, their status of "coefficients" is modified, and they become controls or regulons, according to the context (engineering or life sciences where the problem is set).

Whenever the viability property is concerned, we shall give a name to this phenomenon which seems to be shared by so many systems dealing with living beings: In a loose way, the inertia principle states that the "regulons" of the system are kept constant as long as possible and changed only when viability or inertia is at stake.

The inertia principle provides a mathematical explanation of the emergence of the concept of punctuated equilibrium introduced in paleontology by Nils Eldredge and Stephen J. Gould in 1972.

However, they were anticipated by Darwin himself, who added in the sixth edition of is celebrated book the sentence "and lastly, although each species must have passed through numerous transitional stages, it is probable that the periods, during which each underwent modification, though many and long as measured by years, have been short in comparison with the periods during which each remained in an unchanged condition" (personal communication by Jim Murray).

For instance, this has been documented in paleontology: 
9 Excavations at Lake Turkana Excavations at Kenya's Lake Turkana have provided clear evidence of evolution from one species to another. The rock strata there contain a series of fossils that show every small step of an evolution journey that seems to have proceeded in fits and starts. Examination of more than 3,000 fossils by Peter Williamson showed how 13 species evolved. The record indicated that the animals stayed much the same for immensely long stretches of time. But twice, about two million years ago and then, 700,000 years ago, the pool of life seemed to explode - set off, apparently, by a drop in the lake's water level. Intermediate forms appeared very quickly, new species evolving in 5,000 to 50,000 years, after millions of years of constancy, leading paleontologists to challenge the accepted idea of continuous evolution.

We can translate these biological concepts into precise mathematical definitions. A viability niche of a regulon $u$ is the viability kernel of the system $x^{\prime}(t)=f(x(t), u)$ regulated by the constant regulon $u$. A regulon $u$ is a punctuated equilibrium if and only if its viability niche is non empty. We can prove the existence of "heavy evolutions locking in the viability niche of a punctuated equilibrium" whenever the evolution of the regulon reaches such a punctuated equilibrium on its way, in the sense that it may remain viable in the viability niche forever. The concept of "locking-in" had been introduced in the different field of economics (of innovation), and with other mathematical techniques, for explaining why, once adopted, some technologies, which may look non-optimal in regard of some criterion, are still adopted, whereas some better solutions are not adopted. The same phenomenon appears in biological evolution, and may be explained mathematically by the inertia principle in the paradigm of adaptation to an environment. These are the very considerations which triggered the investigations of what became viability theory at the end of the 1970's.

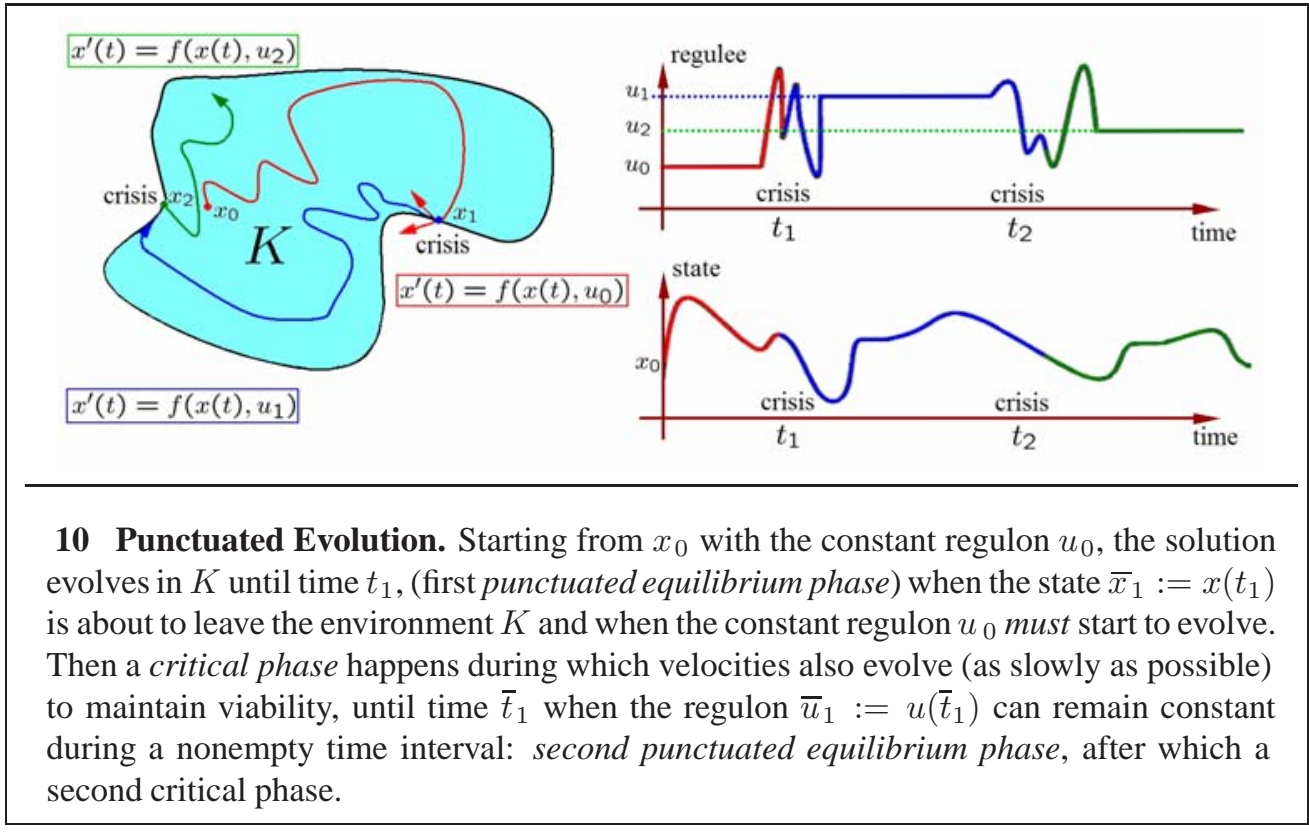




\section{Illustration: Inertia Function}

This is not the place to provide the mathematical details. We rather illustrate these concepts with the simplest possible one dimensional example.

Assume that we have to model a system, the dynamics of which are unknown to us. Hence, we describe our ignorance by an "open" right hand side

$$
x^{\prime}(t)=u(t) \text { where } u(t) \in \mathbb{R}
$$

where the right hand side can be regarded as a regulon or a control. It is tempting to try guessing right hand sides by selecting feedbacks $x \mapsto \widetilde{u}(x)$ and solving

$$
x^{\prime}(t):=\widetilde{u}(x(t))
$$

For instance, the simple examples, besides constant right hands side $\widetilde{u}(x) \equiv u$, are provided byaffine feedbacks defined by $\widetilde{u}(x):=r(b-x)$. The solution starting from $x \in[a, b]$ is equal to $x(t)=e^{-r t} x+b\left(1-e^{-r t}\right)$. It remains viable in the interval $[a, b]$, increases with time with negative acceleration with the norm smaller than or equal to $r^{2}(b-a)$ and converges to the equilibrium $b$ when $t \mapsto+\infty$. Many other examples of right hand sides have been produced, but, for economic or biological systems, we really don't know them, and, in particular, right hand sides producing periodic or cyclic evolutions.

\subsection{The Simplest Example of Inertia Function}

Instead, we can replace our ignorance of the dynamics by some more accessible knowledge, for instance,

$$
\left\{\begin{array}{l}
(i) \quad \text { viability constraints: } x(t) \in K:=[a, b], 0<a<b<+\infty \\
\text { (ii) } \quad \text { inertia threshhold } c \in \mathbb{R}_{+}:\left\|x^{\prime \prime}(t)\right\| \leq c
\end{array}\right.
$$

The above dynamical inequality can be written in the form of the regulated "metasystem"

$$
\begin{cases}(i) & x^{\prime}(t)=u(t) \\ (\text { ii }) & u^{\prime}(t)=v(t) \\ & \text { where }\|v(t)\| \leq y(t)\end{cases}
$$

(where the controls are the accelerations), the equilibria of which are of the form $(x, 0)$ where $x \in[a, b]$.

These simple constraints are enough to deduce many properties of the evolutions governed by the regulated system (3), p.29. We denote by $\mathcal{P}(x, u)$ the set of solutions to differential equation (3) viable in the interval $[a, b]$ such that $x(0)=x$ and $u(0)=x^{\prime}(0)=u$. We introduce the important notion of inertia function defined by

$$
\alpha(x, u):=\inf _{x(\cdot) \in \mathcal{P}(x, u)} \sup _{t \geq 0}\left\|u^{\prime}(t)\right\|=\inf _{x(\cdot) \in \mathcal{P}(x, u)} \sup _{t \geq 0}\left\|x^{\prime \prime}(t)\right\|
$$


It happens that we can characterize it thanks to the concept of viability kernel. Let us introduce for that purpose the auxiliary system (6)

$$
\begin{cases}(i) & x^{\prime}(t)=u(t) \\ (\text { ii }) & u^{\prime}(t)=v(t) \\ (\text { iii }) & y^{\prime}(t)=0 \\ & \text { where }\|v(t)\| \leq y(t)\end{cases}
$$

One can prove that the inertia function is characterized by formula

$$
\alpha(x, u)=\inf _{(x, u, y) \in \operatorname{Viab}_{6}}{ }^{\left(K \times \mathbb{R} \times \mathbb{R}_{+}\right)} y
$$
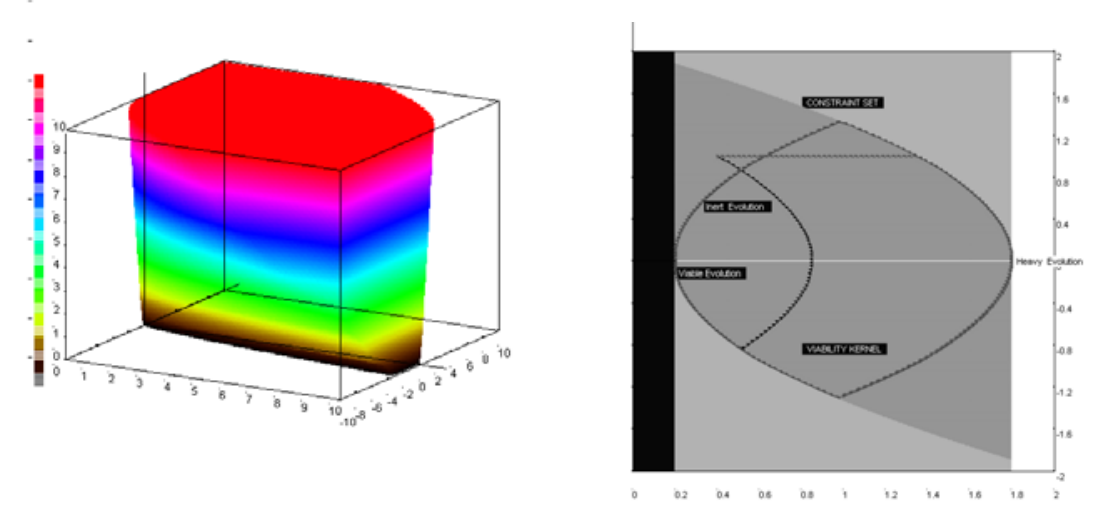

Figure 6.1 Newtonian Inertia Function. [Left.] The Saint-Pierre Viability Kernel Algorithm computes the graph of the inertia function. [Right.] A lower lever set (or section) of the inertia function: Its boundary is the critical zone, over which the state evolves with constant acceleration. The trajectory of an heavy evolution minimizing the velocity of the controls and which stops at equilibrium $b$ is shown.

Therefore, the inertia function inherits all the properties of viability kernel and can be computed thanks to the viability kernel algorithm. In this simple example, we can even provide explicit formulas. One can check for instance the analytical formula of the inertia function:

Lemma 6.2 [Newtonian Inertia Function] The Newtonian inertia function $\alpha$ defined on ]$a, b[\times \mathbb{R}$ is equal to:

$$
\alpha(x, u):=\frac{u^{2}}{2(b-x)} \text { if } u \geq 0 \text { and } \frac{u^{2}}{2(x-a)} \text { if } u \leq 0
$$

Its domain is $\left(\{a\} \times \mathbb{R}_{+}\right) \cup(] a, b\left[\times \mathbb{R}_{+}\right) \cup\left(\{b\} \times \mathbb{R}_{-}\right)$. Hence, from each state-regulon pair $(x, u) \in \operatorname{Dom}(\alpha)$ starts at least one evolution with bounded acceleration, actually, bounded by $\alpha(x, u)$. 
We can also check that the inertia function is the (smallest positive lower semicontinuous) solution to the Hamilton-Jacobi partial differential equation

$$
\forall(x, u) \in] a, b\left[\times \mathbb{R}, \quad \frac{\partial \alpha(x, u)}{\partial x} u-\alpha(x, u)\left|\frac{\partial \alpha(x, u)}{\partial u}\right|=0\right.
$$

We do not need to use this partial differential equation to deduce the properties of the inertia functions which are obtained directly through the viability characterization of the inertial function. Actually, this type of viability characterization holds true for a class of first-order nonlinear partial differential equations.

We associate with the inertia function the inert regulation map $R_{c}$ defined by $R_{c}(x):=$ $\{u \in \mathbb{R}$ such that $\alpha(x, u)=u\}$ and the critical map $\Xi_{c}$ defined by $\Xi_{c}(u):=\{x \in$ $[a, b]$ such that $\alpha(x, u)=c\}$. When $c>0$, the subset $\Xi_{c}(u)$ is called the critical zone of the control $u$ bounded by inertia threshold $c>0$. When $c=0$, the subset $\Xi_{0}(u)$ is called the viability niche of the control $u$. A regulon is a punctuated equilibrium (or, actually, punctuated regulon) if its viability niche $\Xi_{0}(u)$ is not empty.

\section{Lemma 6.3 [Inert Regulation and Critical Maps] We set}

$$
r^{\sharp}(x):=\sqrt{2(b-x)}, r^{b}(x):=\sqrt{2(x-a)} \text { and } R(x):=\left[-r^{b}(x),+r^{\sharp}(x)\right]
$$

The regulation map is equal to

$$
R_{c}(x):=\sqrt{c}\left[-r^{b}(x),+r^{\sharp}(x)\right]=\sqrt{c} R(x)
$$

The critical map $\Xi_{c}(c>0)$ is defined by

$$
\Xi_{c}(u)=b-\frac{u^{2}}{2 c} \text { if } u>0 \text { and } \Xi_{c}(-|u|)=a+\frac{u^{2}}{2 c} \text { if } u<0
$$

The viability niche $\Xi_{0}(u)$ of the regulon $u$ is empty when $u \neq 0$ and equal to $\Xi_{0}(0)=$ $[a, b]$ when $u=0$.

The graph of the regulation map $R_{c}$ associated to the inertia function is limited by the union $\sqrt{c}\left\{-r^{b}(x),+r^{\sharp}(x)\right\}$ of the two graphs of $-\sqrt{c} r^{b}$ below and $\sqrt{c} r^{\sharp}$ above.

\subsection{Heavy Evolutions}

Heavy evolutions with an inertia threshold $c$ are the ones which are viable, satisfy the inertia threshold and which minimize at each instant the norm of the velocity of the regulon. In our case, they minimize at each instant the norm of the acceleration.

We start with regulons with inertia $\alpha(x, u)<c$ strictly smaller than an imposed inertia threshold $c$. We can choose the heavy evolution which is governed by the regulon constant 
equal to $u$ until it reaches the critical zone $\Xi_{c}(u)$ and, next, we shall have to switch the regulon to pilot an evolution until it reaches the equilibrium $(a, 0)$ or $(b, 0)$.

The warning time $\sigma_{c}(x, u) \in \mathbb{R} \cup\{+\infty\}$ is the first instant when evolutions $x_{u}(\cdot)$ starting from $x$ when $x_{u}\left(\sigma_{c}(x, u)\right) \in \Xi_{c}(u)$ reaches the critical zone $\Xi_{c}(u)$ of the regulon $u$.

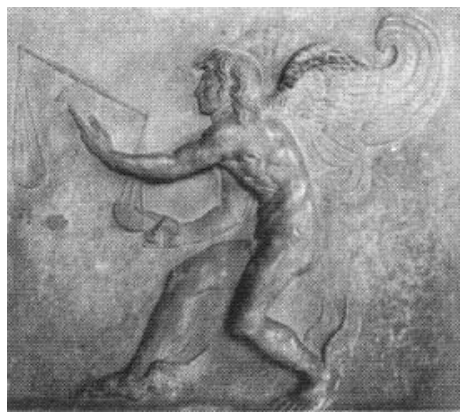

11 [Warning Time or "Kairos".] The concept of warning time is a mathematical translation of the anglo-saxon concept of timing, or the Italian concept of tempismo, modernizing the concept of kairos of classical Greece, meaning propitious or opportune moment.

The ancient Greeks used this qualitative concept of time by opposition to chronos, the quantitative ... chronological time, which can be measured by clocks.

The search of kairos translates the search of decisions taken at the right moment (warning time) rather than static optimal decisions.

Lysippos sculptured a wonderful concrete representation of this very abstract concept (in the museum of Torino).

Lemma 6.3. $\mathrm{p} 31$ implies that solutions $x \pm|u| t$ regulated by constant regulons $\pm|u|$ reach the critical zone at warning time equal to

$$
\sigma_{c}(x, u):=\frac{(b-x)}{|u|}-\frac{|u|}{2 c} \text { if } u>0 \text { and } \sigma_{c}(x, u):=\frac{(x-a)}{|u|}-\frac{|u|}{2 c} \text { if } u<0
$$

because $x+u \sigma_{c}(x, u)=\Xi_{c}(u)=b-\frac{u^{2}}{2 c}($ when $u>0)$.

The inertia function $\alpha$ increases over the evolution under constant regulon $u$ according

$$
\forall t \in\left[0, \frac{(b-x)}{u}-\frac{|u|}{2 c}\right],\left\{\begin{array}{l}
u_{c}(t)=u \\
x_{c}(t):=x+t u \\
\alpha\left(x_{c}(t), u\right)=\frac{u^{2}}{2((b-x)-u t)}
\end{array}\right.
$$

until warning time $\sigma_{c}(x, u)$ when the state reaches $\Xi_{c}(u)$ and the regulon $\alpha_{c}\left(\Xi_{c}(u), u\right)=$ c.

This is the last moment when we have to change the regulon $u$ because, otherwise, either it the regulon remains constant and leaves the environment $[a, b]$ in finite time $\frac{b-x}{|u|}$ $(u>0)$, or, in order to remain viable, it has to violate the inertia threshold strictly before reaching the part $\{b\} \times \mathbb{R}_{+}$of the boundary $\{a, b\} \times \mathbb{R}$ of the environment $[a, b] \times \mathbb{R}$, when it has to leave immediately the environment $[a, b] \times \mathbb{R}$.

When it reaches the critical zone, then the regulon must change with a velocity smaller than or equal to $c$. A general theorem by Marc Quicampoix on the semi-permeable barrier property of the viability kernel implies that actually, the norm of this velocity has to be 
equal to the inertia threshold until it reaches the boundary $\{a, b\} \times \mathbb{R}$ of the environment $[a, b] \times \mathbb{R}$.

In our case, the state-regulon pair evolves according (when $u>0$ ):

$$
\begin{gathered}
\forall t \in\left[\frac{(b-x)}{u}-\frac{|u|}{2 c}, \frac{(b-x)}{u}+\frac{|u|}{2 c}\right] \\
\left\{\begin{array}{l}
u_{c}(t)=u-t c \\
x_{c}(t):=x+u\left(t-\frac{(b-x)}{u}+\frac{|u|}{2 c}\right)-\frac{c}{2}\left(t-\frac{(b-x)}{u}+\frac{|u|}{2 c}\right)^{2} \\
\alpha\left(x_{c}(t), u\right)=c
\end{array}\right.
\end{gathered}
$$

until warning time $\frac{(b-x)}{u}+\frac{|u|}{2 c}($ when $u>0)$.

Heavy evolution display a behavior which is reminiscent of the concept of Tychonov slow and fast evolutions, where the the regulon would play the role of the slow variable and the state the role of the fast variable (see for instance [17, 18, Lobry, Sari \& Touhami] among a huge literature). However the slowness is not dictated by an a priori coefficient $\varepsilon$ converging to 0 , but is either very slow, indeed, constant, or as slow as possible with a velocity imposed by the inertia threshold. The state evolves according to the heavy control, with no other limit on its velocity. The paper [16, Lobry \& Sari] comes back to this question by using nonstandard analysis.

\subsection{Heavy Viability Cycles and Hysterons}

Let us choose an inertia threshold $c$ and assume that $|u| \leq \sqrt{c} \sqrt{b-a}$.

Instead of choosing the heavy evolution which remains at one of the equilibria $(a, 0)$ or $(b, 0)$ forever by switching the acceleration (velocity of the regulon) to 0 , we continue the evolution by keeping the acceleration $-c$ or $+c$ as long as possible, and then, switch again to 0 , again, as long as it is possible to obey viability and inertia constraints.

Recall that the critical map is equal to

$$
\Xi_{c}(u)=b-\frac{u^{2}}{2 c} \text { if } u>0 \text { and } \Xi_{c}(-|u|)=a+\frac{u^{2}}{2 c} \text { if } u<0
$$

We set

$$
x^{\star}:=\frac{b-a}{2} \text { and } u^{\star}:=\sqrt{b-a}
$$

The two values are equal if $u=\sqrt{c} u^{\star}$ where we set $u^{\star}:=\sqrt{b-a}$. We observe that $x^{\star}:=\Xi_{c}\left(\sqrt{c} u^{\star}\right)=\frac{b-a}{2}$ does not depend on $c$.

Hence, by 9, 192 the warning time being equal to

$$
\sigma_{c}(x, u):=\frac{(b-x)}{|u|}-\frac{|u|}{2 c} \text { if } u>0 \text { and } \sigma_{c}(x, u):=\frac{(x-a)}{|u|}-\frac{|u|}{2 c} \text { if } u<0
$$

we deduce that

$$
\sigma_{c}(a, u)=\frac{(b-a)}{|u|}-\frac{|u|}{2 c} \text { and } \sigma_{c}\left(a+\frac{u^{2}}{2 c}\right)=\frac{(b-a)}{|u|}-\frac{|u|}{c}
$$


Recall that $\frac{|u|}{c}$ is the time needed to reach

$$
(b, 0) \text { from }\left(\Xi_{c}(u), u\right) \quad \text { or }(a, 0) \text { from }\left(\Xi_{c}(-|u|),-|u|\right) .
$$

If $u:=\sqrt{c} u^{\star}$, then $\frac{\sqrt{c}\left|u^{\star}\right|}{c}=\frac{\sqrt{b-a}}{\sqrt{c}}$.

We define the heavy hysteresis cycle $\left(x_{h}(\cdot), u_{h}(\cdot)\right)$ of cycle $2\left(\frac{b-a}{|u|}+\frac{|u|}{c}\right)$ starting at $\left(\Xi_{c}(-|u|), u\right)$ with $u>0$ where $\Xi_{c}(-|u|):=a+\frac{u^{2}}{2 c}$ in the following way:

1) The state-regulon pair $\left(x_{h}(\cdot), u_{h}(\cdot)\right)$ starts from $\left(\Xi_{c}(-|u|), u\right)$ by taking the velocity of the regulon equal to 0 . It remains viable on the time interval $\left[0, \frac{b-a}{|u|}-\frac{|u|}{c}\right]$ until it reaches the state-regulon pair $\left(\Xi_{c}(u), u\right)$ where $\Xi_{c}(u):=b-\frac{u^{2}}{2 c}$ because $\sigma_{c}\left(a+\frac{u^{2}}{2 c}\right)=\frac{(b-a)}{|u|}-\frac{|u|}{c}$.

2) The state-regulon pair $\left(x_{h}(\cdot), u_{h}(\cdot)\right)$ starts from $\left(\Xi_{c}(u), u\right)$ at time $\frac{b-a}{|u|}-\frac{|u|}{c}$ by taking the velocity of the regulon (acceleration) equal to $-c$.

- It ranges over the graph of $\sqrt{c} r^{\sharp}(x)$ on the time interval $\left[\frac{b-a}{|u|}-\frac{|u|}{c}, \frac{b-a}{|u|}\right]$ until it reaches the state-regulon pair $(b, 0)$. The heavy evolution would remain at this equilibrium forever with an acceleration equal to 0 .

- However, for defining the heavy hysteresis cycle, we assume that we keep the acceleration equal to $-c$. Hence the state-regulon pair $\left(x_{h}(\cdot), u_{h}(\cdot)\right)$ ranges over the graph of $\sqrt{c} r^{\sharp}(x)$ on the time interval $\left[\frac{b-a}{|u|}, \frac{b-a}{|u|}+\frac{|u|}{c}\right]$ until it reaches the state-regulon pair $\left(\Xi_{c}(u),-|u|\right)$.

3) The state-regulon pair $\left(x_{h}(\cdot), u_{h}(\cdot)\right)$ starts from $\left(\Xi_{c}(u),-|u|\right)$ at time $\frac{b-a}{|u|}+\frac{|u|}{c}$ by taking the velocity of the regulon equal to 0 . It remains viable on the time interval $\left[\frac{b-a}{|u|}+\frac{|u|}{c}, 2 \frac{b-a}{|u|}\right]$ until it reaches the state-regulon pair $\left(\Xi_{c}(-|u|),-u\right)$.

4) The state-regulon pair $\left(x_{h}(\cdot), u_{h}(\cdot)\right)$ starts from $\left(\Xi_{c}(-|u|),-u\right)$ at time $2 \frac{b-a}{|u|}$ by taking the velocity of the regulon equal to $+c$.

- It ranges over the graph of $-\sqrt{c} r^{b}(x)$ on the time interval $\left[2 \frac{b-a}{|u|}, 2 \frac{b-a}{|u|}+\frac{|u|}{c}\right]$ until it reaches the state-regulon pair $(a, 0)$. The heavy evolution would remain at this equilibrium forever with an acceleration equal to 0 .

- However, for defining the heavy hysteresis cycle, we assume that we keep the acceleration equal to $+c$. Hence the state-regulon pair $\left(x_{h}(\cdot), u_{h}(\cdot)\right)$ ranges over the graph of $+\sqrt{c} r^{b}(x)$ on the time interval $\left[2 \frac{b-a}{|u|} c+\frac{|u|}{c}, 2\left(\frac{b-a}{|u|}+\frac{|u|}{c}\right)\right]$ until it reaches the state-regulon pair $\left(\Xi_{c}(-|u|), u\right)$. 
This ends the heavy hysteresis cycle.

Heavy hysteresis cycles are governed by three velocities of regulons only, $-c, 0$ and $c$, which can be regarded as "meta-regulons". They provide an example of "quantized" evolutions, governed by a combination (called amalgam) of a finite number of (meta) regulons only. Quantization is a recent issue in control theory, where, instead of computing the complicated feedback regulating viable evolutions, the question arises to achieve the regulation of viable evolutions with a finite number of controls or feedbacks.

This number can be reduced to two (meta) regulons in the limiting case $u:=\sqrt{c} u^{\star}$ and when $\Xi_{c}\left(\sqrt{c} u^{\star}\right)=\Xi_{c}\left(\sqrt{c} u^{\star}\right)=: x^{\star}$. In this case, heavy hysteresis cycles are called inert hysteresis cycle $x_{h}(\cdot)$ (of cycle $\frac{4 u^{\star}}{\sqrt{c}}$ ) in the following way:

1) The state-regulon pair $\left(x_{h}(\cdot), u_{h}(\cdot)\right)$ starts from $\left(x^{\star}, \sqrt{c} u^{\star}\right)$ at time 0 by taking the velocity of the regulon equal to $-c$. It ranges over the graph of $\sqrt{c} r^{\sharp}$ on the time interval $\left[0, \frac{u^{\star}}{\sqrt{c}}\right]$ until it reaches the equilibrium $(b, 0)$ and next, keeping the velocity of the regulon equal to $-c$, it ranges over the graph of $-\sqrt{c} r^{\sharp}$ on the time interval $\left[\frac{u^{\star}}{\sqrt{c}}, \frac{2 u^{\star}}{\sqrt{c}}\right]$ until it reaches the state-regulon pair $\left(x^{\star},-\sqrt{c} u^{\star}\right)$.

2) The state-regulon pair $\left(x_{h}(\cdot), u_{h}(\cdot)\right)$ starts from $\left(x^{\star},-\sqrt{c} u^{\star}\right)$ at time $\frac{2 u^{\star}}{\sqrt{c}}$ by taking the velocity of the regulon equal to $+c$. It ranges over the graph of $-\sqrt{c} r^{b}$ on the time interval $\left[\frac{2 u^{\star}}{\sqrt{c}}, \frac{3 u^{\star}}{\sqrt{c}}\right]$ until it reaches the equilibrium $(a, 0)$ and next, keeping the velocity of the regulon equal to $+c$, it ranges over the graph of $+\sqrt{c} r^{b}$ on the time interval $\left[\frac{3 u^{\star}}{\sqrt{c}}, \frac{4 u^{\star}}{\sqrt{c}}\right]$ until it reaches the state-regulon pair $\left(x^{\star}, \sqrt{c} u^{\star}\right)$.

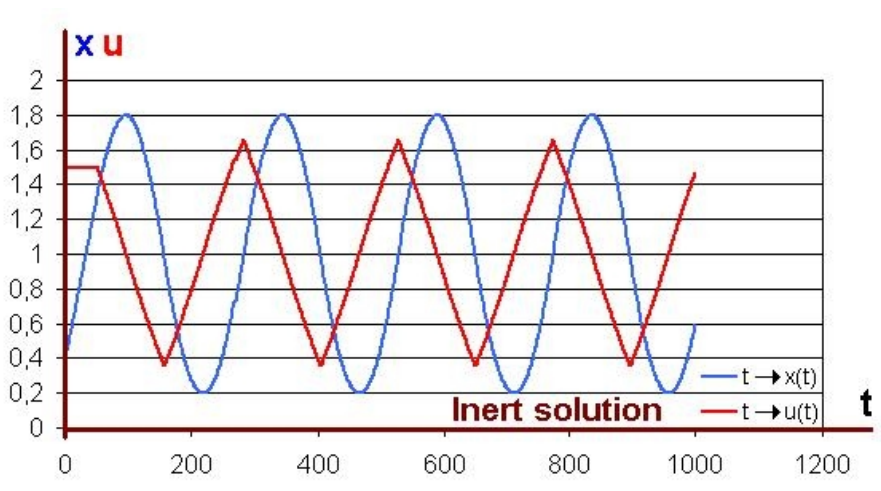

12 Evolutions of Inert Evolutions and their Controls. The Viability Kernel Algorithm computes both the graphs of the smooth inert evolution (in blue) and of its regulon (in red) are plotted. The velocity of the regulon oscillates from $+u^{\star}$ to $-u^{\star}$. The evolution is then cyclic, alternatively increasing and decreasing from $a$ to $b$.

The trajectories of the inert evolutions can be computed with the viability kernel algorithm (without using the analytical formulas specific to this example): 


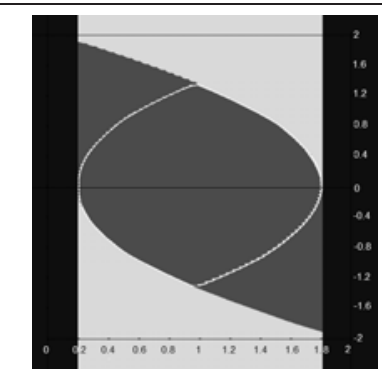

The Inert Hysteresis Loop. The Viability Kernel Algorithm computes the the graph of the regulation map $R_{c}$ (which is a viability kernel) and the inert hysteresis loop when $u:=\sqrt{c(b-a)}$, where the Inert Hysteresis Loop is governed by two metacontrols $+c$ and $-c$.

The evolutions goes from a to $b$ by one route and from $b$ to a by another route.

Observe that the state oscillates from $a$ to $b$ back and forth, but by two different paths. This is a hysteresis loop:

13 [Hysteresis Loops and Hysterons] James Ewin, a Scottish physicist discovered and coined the word hysteresis meaning lagging behind in classical Greek. This polysemous word is used in many different fields, including in mathematics, where several mathematical translations have been observed (among which [21] Visintin]'one, related to subsets invariant with respect to affine time scaling). We use it here as an engine, called hysteron, producing hysteresis loops or hysteresis cycles: When the evolution of the state of a system with hysteresis is plotted on a graph against the applied force (in our case, the regulon), the resulting curve has the shape of a loop.

So, the heavy viability oscillator produces the following hysteron:

Lemma 6.4 [The Heavy Hysteron] Let us assume that $|u| \leq \sqrt{c} \sqrt{b-a}$. The heavy hysteron is the set-valued map $\Phi_{\text {Heavy }}$ defined by

$$
\Phi(x)=\left\{-\min \left(u, \sqrt{c} r^{b}(x), \sqrt{c} r^{\sharp}(x)\right), \min \left(u, \sqrt{c} r^{b}(x), \sqrt{c} r^{\sharp}(x)\right)\right\}
$$

Its graph is the heavy hysteresis loop in the state-regulon space (actually, the phase space). Its cycle is equal to $2\left(\frac{b-a}{|u|}+\frac{|u|}{c}\right)$.

When regulated by positive regulons, the state goes from $a$ to $b$ and the state-regulon pair ranges over the graph of $x \mapsto \min \left(u, \sqrt{c} r^{b}(x), \sqrt{c} r^{\sharp}(x)\right)$ whereas, when regulated by negative regulons, the state goes from $b$ to $a$, and the state-regulon pair ranges over the graph of $x \mapsto-\min \left(u, \sqrt{c} r^{b}(x), \sqrt{c} r^{\sharp}(x)\right)$. The evolution $t \mapsto\left(x_{h}(t), u_{h}(t)\right)$ is cyclic of cycle $2\left(\frac{b-a}{|u|}+\frac{|u|}{c}\right)$.

In the case when $u:=\sqrt{c} u^{\star}$, figure 6.3), $\mathrm{p} 36$ provides the hysteresis loop of the heavy hysteron computed by the viability kernel algorithm. 
14 This very simple mathematical metaphor implies that two excitatory/inhibitory simple mechanism of a DNA site with bounds on the quantities and their accelerations are sufficient to explain the production of an isolated protein increasing up to a given viability bound and then, decreasing to disappear and being produced again according to a clock, the cyclicity of which is concealed in this very simple viability oscillator, triggering a biological clock.

\section{Conclusion}

We presented a quite long answer to the question of the relevance of present day mathematical and illustrated it by the concepts of inertia principe, heavy evolutions and heavy cycles and hysterons based only on viability constraints and inertia threshold in the simplest situation.

Needless to say, the results illustrated by this example are quite general, and apply to a large class of nonlinear regulated systems (see [5, Aubin \& Saint-Pierre] for instance). Curiously, and unexpectedly, the very concept of viability kernel describing the basic viability problem played also a crucial role to characterize the inertia function. This function helped us to give a precise statement of the concept of inertia principle as well as the simplest evolutions satisfying it, the heavy evolutions. Starting with any regulated system (and not only the simple system $x^{\prime}(t)=u(t)$ ), viability constraints and inertia threshold, the same story remains true and we can associate heavy viability cycles driven by the regulated system. Heavy evolutions lock-in the viability niche of a regulon in the sense that whenever it enters this viability niche, the evolution may (and not must if the system is not deterministic) remain in it forever. We do not need to assume that the system governing the evolution of the state is periodic for providing cyclic evolutions.

This is an illustration of the viability tools which are meant to enrich the panoply of those diverse and ingenious techniques set out by the study of dynamical systems since the pioneering works of Alexander Lyapunov and Henri Poincaré more than one century ago. Most of them were motivated by physics and mechanics, not necessarily designed to adaptation problems to environmental or viability constraints. Viability theory incorporates some mathematical features of uncertainty without statistical regularity, deals not only with intertemporal optimality but also with viability and decisions taken at warning time (kairos) instead of "optimal" ones. Viability techniques are also geometric in nature, but they do not require smoothness properties usually assumed in differential geometry. They not only deal with asymptotic behavior, but also and mainly with transient evolutions and capturability of targets in finite or prescribed time. They are global instead of local, and really nonlinear since they bypass linearization techniques of the dynamics around equilibria, for instance. They bring other lights to the decipherability of complex, paradoxical and strange dynamical behaviors by providing other types of mathematical results and algorithms. And above all, they have been motivated by dynamical systems arising in issues involving living beings, as well as networks of systems (or organizations, organisms). 
In a nutshell, and for the time, viability theory investigates evolutions

1) in continuous time, discrete time, or an "hybrid" of the two when impulses are involved,

2) constrained to adapt to an environment,

3) evolving under contingent, stochastic or tychastic uncertainty (where "tyches" replace probabilistic "events"),

4) using for this purpose regulons (regulation regulons), subsets of regulons, and in the case of networks, connectionnist matrices,

5) regulated by feedback laws (static or dynamic) that are then "computed" according to given principles, such as the inertia principle, intertemporal optimization, etc.,

6) co-evolving with their environment (mutational and morphological viability),

7) and corrected by introducing adequate regulons (viability multipliers) when viability or capturability is at stakes.

Although viability theory has been designed and developed for studying the evolutions of uncertain systems confronted to viability constraints arising in socio-economic and biological sciences, as well as in control theory, it had also been used as a mathematical tool for analyzing the local and asymptotic behavior of control systems, either in continuous or discrete time, even as "wild" yet deterministic systems such as the famous Lorenz system or the complex quadratic map, as well as an efficient tool for solving systems of first-order partial differential equations.

After years of study of various problems of different kinds, motivated from robotics (and animat theory), game theory, economics, neuro-sciences (see for instance [2, Aubin]), biological evolution and, unexpectedly, from financial mathematics, these few relevant traits common to all these problems were uncovered, after noticing the common features of the proofs and algorithms. This history is a kind of mathematical striptease, the modern version of what Parmenides and the pre-Socratic Greeks called a-letheia, the discovering, un-veiling of the world that surrounds us. This is nothing else than the drive to "abstraction", isolating, in a given perspective, the relevant information in each concept and investigate the interplay between them. Indeed, one by one, slowly and very shyly, the required properties of the regulated system were taken away. If there is one domain where mankind made some progress, it is this long march to abstraction:

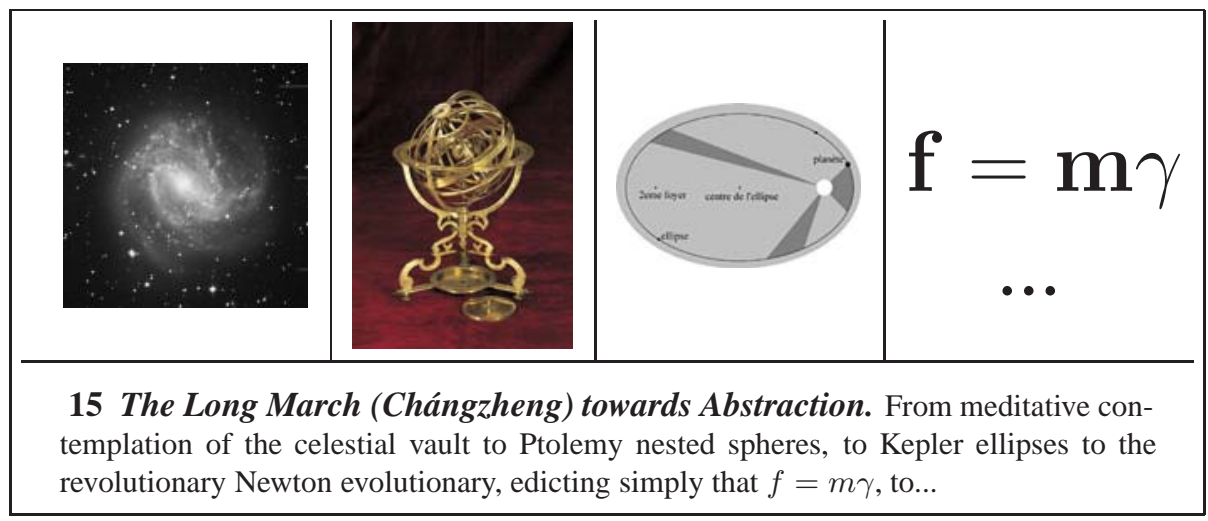




\section{References}

[1] Aubin J.-P. (1991) Viability theory, Birkhäuser

[2] Aubin J.-P. (1996) Neural networks and qualitative physics: a viability approach, Cambridge University Press

[3] Aubin J.-P. (1997) Dynamic economic theory: a viability approach, Springer-Verlag

[4] Aubin J.-P. (2000) Mutational and morphological analysis: tools for shape regulation and morphogenesis, Birkhäuser

[5] Aubin J.-P. \& Saint-Pierre P. (2006) Guaranteed Inertia Functions in Dynamical Games, International Game Theory Review (IGTR), 8, 185 - 218

[6] Aubin J.-P. (2008) La mort du devin, l'émergence du démiurge. Essai sur la contingence et la viabilité des systèmes, Éditions Beauchesne

[7] Aubin J.-P., Bayen A., Bonneuil N. \& Saint-Pierre P. (2009) Viability, Control and Games: Regulation of Complex Evolutionary Systems Under Uncertainty and Viability Constraints, Springer-Verlag

[8] Aubin J.-P. \& Cellina A. (1984) Differential inclusions, Springer-Velag, Grundlehren der math. Wiss. \# 264

[9] Aubin J.-P. \& Frankowska H. (1990) Set-valued analysis, Birkhäuser

[10] Aubin J.-P.\& Lesne A. (2006) Analyse morphologique et mutationnelle: des outils pour la morphogenèse, in Morphogenèse, Bourgine P. \& Lesne A. Edts, Belin, 162-177

[11] Fruchard A. \& Schäfke R. (2008)Sur le retard à la bifurcation, This volume

[12] Françoise J.-P. (2005) Oscillations en biologie. Analyse qualitative et modèles, Springer

[13] Lobry C. (1992) A propos du sens des textes mathématiques, un exemple : la théorie des "bifurcations dynamiques", Annales Institut Fourier, 42, 327-351.

[14] Lobry C. (2008) La méthode des élucidations successives, This volume 327-351.

[15] Lobry C. \& Sari T. (2008) La modélisation de la persistance en écologie, Premier congrès de la société marocaine de mathématiques appliquées,

[16] Lobry C. \& Sari T. (2008) Nonstandard Analysis and representation of reality, International J. Control, 81, 519-536

[17] Lobry C., Sari T. \& Touhami S. (1998) On Tykhonov's theorem for convergence of solutions of slow and fast systems, Electron. J. Differential Equations, 22

[18] Lobry C., Sari T. \& Touhami S. (1999) Fast and Slow Feedback in Systems Theory, Journal of Biological Systems, 3, 307-331

[19] Monod J. (1971) Le hasard et la nécessité, Le Seuil

[20] Murray J. (1990) Theoretical Biology, Springer-Verlag

[21] Visintin A. (1994) Differential Models of Hysteresis, Berlin, Springer, 1994.

[22] Wigner E.P. (1960) The unreasonable effectiveness of mathematics in the natural sciences, Communications in Pure and Applied Mathematics, 13, 1-14

[23] Winfree A. (1994) Les horloges de la vie, Belin 